\title{
Do drivers change their manual car-following behaviour after automated car-following?
}

\author{
Tyron Louw ${ }^{1}$ (1) $\cdot$ Rafael Goncalves $^{1} \cdot$ Guilhermina Torrao ${ }^{1} \cdot$ Vishnu Radhakrishnan $^{1} \cdot$ Wei Lyu $^{1,2}$. \\ Pablo Puente Guillen ${ }^{3} \cdot$ Natasha Merat ${ }^{1}$
}

Received: 3 July 2020 / Accepted: 21 November 2020

(c) The Author(s) 2020

\begin{abstract}
There is evidence that drivers' behaviour adapts after using different advanced driving assistance systems. For instance, drivers' headway during car-following reduces after using adaptive cruise control. However, little is known about whether, and how, drivers' behaviour will change if they experience automated car-following, and how this is affected by engagement in non-driving-related tasks (NDRT). The aim of this driving simulator study, conducted as part of the H2020 L3Pilot project, was to address this topic. We also investigated the effect of the presence of a lead vehicle during the resumption of control, on subsequent manual driving behaviour. Thirty-two participants were divided into two experimental groups. During automated car-following, one group was engaged in an NDRT (SAE Level 3), while the other group was free to look around the road environment (SAE Level 2). Both groups were exposed to Long (1.5 s) and Short (.5 s) Time Headway (THW) conditions during automated car-following, and resumed control both with and without a lead vehicle. All post-automation manual drives were compared to a Baseline Manual Drive, which was recorded at the start of the experiment. Drivers in both groups significantly reduced their time headway in all post-automation drives, compared to a Baseline Manual Drive. There was a greater reduction in THW after drivers resumed control in the presence of a lead vehicle, and also after they had experienced a shorter THW during automated car-following. However, whether drivers were in L2 or L3 did not appear to influence the change in mean THW. Subjective feedback suggests that drivers appeared not to be aware of the changes to their driving behaviour, but preferred longer THWs in automation. Our results suggest that automated driving systems should adopt longer THWs in car-following situations, since drivers' behavioural adaptation may lead to adoption of unsafe headways after resumption of control.
\end{abstract}

Keywords Automated vehicles $\cdot$ Behavioural adaptation $\cdot$ Car-following $\cdot$ Driver behaviour $\cdot$ Headway

\section{Introduction}

Advanced Driver Assistance Systems (ADAS), such as Adaptive Cruise Control (ACC) or Lane-Keeping Support (LKS) systems, have become a common feature of modern vehicles. Although the principal purpose of implementing these systems in vehicles is to increase driver and

Tyron Louw

t.1.louw@leeds.ac.uk

1 Institute of Transport Studies, University of Leeds, Leeds LS2 9JT, UK

2 School of Business Administration, Northeastern University, Shenyang, China

3 Toyota Motor Europe NV/SA, Hoge Wei 33, 1930 Zaventem, Belgium road safety, human factors studies, investigating the effect of ADAS on driver behaviour, have shown mixed results. For example, lab-based studies have shown that ACC use reduces drivers' workload (de Winter et al. 2014), but also results in reduced minimum time headway (Hoedemaeker and Brookhuis 1998), and delayed reactions to hazardous events, when compared to manual driving (Larsson et al. 2014; Rudin-Brown and Parker 2004; Shen and Neyens 2017).

Vehicle manufacturers are now striving towards the implementation of more advanced Automated Driving Systems (ADS), where lateral and longitudinal assistances are combined to manage moment-to-moment vehicle control. Examples include SAE Level 2 (L2), partial automation (SAE 2018), which already exists in some high-end brands, where drivers remain responsible for the driving task, and 
are required to monitor the vehicle's performance and external road environment. At the next level of automation, Level 3 (L3), or conditional automation, drivers are allowed to engage in non-driving-related tasks (NDRT), but are still required to be available to resume control, when requested. In terms of understanding the human factors implications of these higher levels of automation, to date, the greatest level of research interest has been on the time it takes drivers to resume control from automation, and how this affects performance and safety (Merat et al. 2014; Eriksson and Stanton 2017), especially on approach to critical situations (Louw et al. 2017b; Zhang et al. 2019). However, less is known about how/if the longer term effects of automation engagement affects drivers' subsequent manual driving performance, and whether this is influenced by engagement in NDRTs (Metz et al. 2020). Since ADS are not, yet, capable of managing all existing road and environmental conditions, the need for drivers' intermittent resumption of control from ADS is likely, for the foreseeable future. Therefore, in addition to understanding the instant effects of such resumptions of control on performance, for collision avoidance and hazard perception, it is also important to assess how they affect less critical driving scenarios, and whether they influence drivers' already well-established behaviours during manual vehicle control (behavioural adaptation). The aim of this study was to consider one such scenario, where ADS engagement was interrupted following a number of car-following scenarios. In particular, we wanted to explore if drivers' preferred "baseline" headway to a lead vehicle was affected after exposure to one of two different headways during automated car-following in L2 and L3. In addition, we investigated if monitoring the environment, and therefore seeing the headway maintained by the ADS (L2), resulted in a different subsequent manual car-following behaviour, compared to situations which did not require driver monitoring (L3). Before describing the methods used, the next sections provide a short overview of car-following and behavioural adaptation research.

\subsection{Car-following situations}

Rear-end collisions are the most common type of collision, accounting for $30.2 \%$ of all collisions in the US (National Highway Traffic Safety Administration [NHTSA] 2009). Car-following refers to a driver's behaviour while following a lead vehicle longitudinally, and is a pre-cursor to rear-end collisions. Car-following is a common characteristic of routine, high-density traffic conditions, and, therefore, safe carfollowing can be considered an important element of safe driving (Saifuzzaman and Zheng 2014). Previous studies have highlighted the role of a number of factors that contribute to rear-end collisions, based on driver, vehicle, roadway, and environmental characteristics. Overall, the predominant factors contributing to rear-end collisions include drivers' failure to perceive and/or react to a lead vehicle's actions, and close car-following behaviour (Dingus et al. 2006).

There are various means by which drivers' vehicle control during car-following can be assessed, including the absolute value and variance of speed, lane position, time, or distance measures. Time headway (THW) is a widely used indicator in this context, used to estimate the criticality of a particular traffic scenario, and is defined as the elapsed time between a following vehicle passing over the same point on the roadway passed by a lead vehicle (Evans 1991). THW is a trade-off between safety and efficiency, where drivers tend to fluctuate their THW within a 'safe boundary' of minimum and maximum THW (Boer 1999). Drivers' THW during carfollowing ranges between 1.2 and $2.5 \mathrm{~s}$ (Siebert et al. 2017; Loulizi et al. 2019); however, this value may vary depending on vehicle speed (Loulizi et al. 2019; Vogel 2003), traffic flow (Salter 1974), and road environment (Vogel 2002). For example, the relationship between THW and vehicle speed is not linear, but follows a right-skewed, bell-shaped curve, sharply increasing up to a particular tipping point, and then gradually decreasing with increase in speed (Niels 2019; Brackstone, Waterson, and McDonald 2009). Moreover, while the threshold for determining car-following events in highway settings is typically between 2.5 and $4 \mathrm{~s}$ (Evans and Wasielewski 1983; Loulizi et al. 2019), Vogel (2002) showed that, in urban environments, it could be as high as $6 \mathrm{~s}$, owing to the relatively lower speeds in those settings.

The adopted THW may also vary depending on driving style, and perceptual-motor abilities of the individual driver (Edie 1961). For example, Van Winsum (1998) showed that the time headway drivers adopt is partly the result of their braking performance, and perceptual-motor skills, where drivers with a smaller preferred time headway tend to be better at tuning their braking performance to the requirements of the situation. Itkonen and Lehtonen (2020) argue that this also interacts with the level of mental effort drivers are willing to invest in the car-following task, where, according to Pekkanen, Lappi, Itkonen and Summala (2017), drivers who prefer to allocate less attention to the road ahead and driving task, and more to other activities, tend to keep longer time headways to the leading vehicle. Considering these results are based on behaviour in manual driving, it would be interesting to investigate what THW drivers prefer, when engaged in other activities during automated driving.

\subsection{Behavioural adaptation}

In the traffic context, Behavioural Adaptation (BA) can be defined as, 'Any change of driver, traveller, and travel behaviours that occurs following user interaction with a change to the road traffic system, in addition to those behaviours 
specifically and immediately targeted by the initiators of the change' (Kulmala and Rämä 2013).

Early models of BA focused on risk compensation, and risk homeostasis, as competing accounts for how, and why, BA occurs. Wilde (1994), for example, views BA as a form of risk compensation, where people (or drivers) change their behaviour to compensate for changes in perceived risk. The risk homeostasis account, on the other hand, holds that drivers do not necessarily compensate for risk, but more likely attempt to match a subjective target (or optimal) level of risk (Trimpomp 1996), by, for example, balancing fluency (e.g., speed) with safety. Both accounts, however, suggest that BA occurs under a perception-evaluation process, with changes in behaviour governed by feedback, based on individual capabilities, and situational circumstances. For example, a driver may speed more on a quiet road, but the extent of this increased speed may depend on their propensity to take risks. Contemporary models of BA, while still lacking agreement on the underlying mechanisms of BA, have placed less emphasis on 'risk', and more significance on the role of emotions, and feelings, in an individual's decisionmaking. It is also acknowledged that BA is influenced by drivers' experience with, and trust in, a system, as well as their mental model of system functionality, and performance (Smiley, 2000; Rudin-Brown and Noy 2002).

Some behavioural adaptations may result in positive outcomes; for example, repeated use of intelligent warning systems has been shown to improve drivers' ability to perceive risks in the environment (Rudin-Brown and Jamson 2013). However, there may also be negative behavioural adaptations, perhaps not anticipated by the designers of the system. For example, various authors have reported that drivers with Adaptive Cruise Control (ACC) engaged were slower to react in critical traffic situations, compared to those without ACC (Nilsson 1995; Larsson, Kircher and Hultgren 2014; Piccinini et al., 2014; de Winter, Happee, Martens and Stanton 2014). In addition, Rudin-Brown and Parker (2004) found that after using ACC, high-sensation seeking drivers reacted to a safety-relevant brake light detection task later, and had more impaired lane-keeping performance, compared to low sensation-seeking drivers. Drivers are also found to adopt shorter time headways in car-following situations and increase their mean speed after using ACC (Hoedemaeker and Brookhuis 1998). In contrast, Piccinini et al. (2014) report that experienced ACC users have longer headways and lower speeds, while Stanton, Young and McCaulder (1997) found that ACC use resulted in no changes to speed or headway. While there is extensive research on how drivers' behaviour adapts after using various ADAS (Sullivan, Flannagan, Pradhan and Bao 2016), understanding adaptation to ACC is most relevant here, because it involves the automatic management of longitudinal control, including that used in car-following situations. In this study, we build on the literature on ACC and BA, to consider the effect of higher levels of automation, where the vehicle also performs lateral control and drivers may be engaged in non-drivingrelated tasks.

\subsection{BA and automated driving}

Drivers' BA to vehicle automation may occur both during automated driving, and during any sections of manual driving, performed after, or between periods of, automated driving. For example, studies have shown that, compared to manual driving, drivers reduce their visual attention to the forward path (Louw and Merat 2017), and increase their engagement in non-driving-related tasks (NDRT) (Carsten et al. 2012), during automated driving, especially with higher levels of automation. Such activities normally lead to missed or delayed response to safety-related cues from the ADS, or the driving environment, during the resumption of control (cf. Louw et al. 2017a; Victor et al. 2018). However, it is currently unclear whether this reduced attention to the road during automated car-following reduces drivers' following distance to the lead vehicle in subsequent manual driving conditions. For example, there is evidence from the aviation domain that aircraft pilots become more willing to take risks after flying in autopilot mode (Lyons et al. 2016), which might suggest that car drivers may be willing to adopt shorter headways following the engagement of automation. Understanding if the headway adopted by the automation influences this behaviour is also relevant.

In terms of adaptation of drivers' behaviour during post-automation manual driving, Skottke et al. (2014) and Eick and Debus (2005), showed that drivers reduced their time headway after being decoupled from highly automated driving. Therefore, it appears that the use of ADS may change the boundary of acceptable risk that drivers are willing to accept during automated driving. However, it is not clear if the length of THW adopted by the ADS (e.g., short versus long THWs) influences drivers' following behaviour, or if this is affected by whether or not drivers are attentive to the ADS behaviour. Research has shown that when an individual uses an automated system for a prolonged period, they tend to develop inappropriately high levels of trust in the system, leading to complacency (Lee and Moray 1994). It is reasonable to assume, therefore, that following prolonged automated car-following at short headways, drivers who adopt lower headways than they are normally comfortable with in manual driving, are in danger of compromising their ability to avoid rear-end collisions, if they adopt a new, shorter, headway. 


\subsection{Aims of the current study}

The main aim of this driving simulator study, conducted as part of the H2020-funded L3Pilot project, was to understand whether experiencing automated car-following influences drivers' subsequent manual car-following behaviour. As reported above, the two predominant factors contributing to rear-end collisions are a driver's failure to perceive and/or react to a lead vehicle's action, likely to be exacerbated by close car-following behaviour (Dingus et al. 2006). However, these two factors have not yet been systematically investigated in the context of BA and vehicle automation. Therefore, to address the first aim of this study, an urban car-following scenario was created, where all drivers were exposed to one of two THW conditions ( $0.5 \mathrm{~s}$ vs $1.5 \mathrm{~s})$ maintained by a highly automated vehicle. We assessed whether exposure to these two THWs changed drivers' adopted THW in a subsequent manual car-following situation, compared to their initial THW in manual driving, before automation was experienced. These THW parameters were based on the 25th and 75th percentile of a driver behaviour model, based on naturalistic driving studies, which incorporate drivers' instantaneous aggressiveness during car-following scenarios (Niels, Edoardo, Florent and Clément 2019). Our aim was to expose drivers to two fairly 'aggressive' automated carfollowing scenarios. A $1.5 \mathrm{~s}$ THW has been used in other studies (cf. de Waard et al. 1999; Lyu et al. 2019; Heikoop, de Winter, van Arem, and Stanton 2019). We avoided longer THWs, to ensure that drivers did not feel too disconnected from the lead vehicle. The shorter $0.5 \mathrm{~s}$ THW was chosen to allow an observable comparison in behaviour with this headway. We hypothesised that, overall, drivers will reduce their THW in manual car-following after experiencing automated car-following, but that this reduction will be greater after experiencing the shorter THW.

The second aim of this study was to understand how engagement with the driving task during automated carfollowing influenced whether drivers changed their THW in subsequent manual car-following. We hypothesised that drivers in L2 automation, who are expected to continuously monitor the road environment, would be more susceptible to changing their THW after automated car-following, than drivers in L3, who were encouraged to look away from the road environment, and were perhaps not aware of the two automated headways.

Table 1 Participant demographics information
Given the emphasis on personal characteristics in determining susceptibility to BA, and driving style, more generally (Itkonen and Lehtonen 2020), we also investigated whether changes in THW would co-vary with drivers' selfreported traits, including sensation seeking (Arnett 1994), traffic locus of control (Özkan and Lajunen 2005), and driver style questionnaire (French, West, Elander and Wilding 1993). Drivers with an external LOC and who scored high on the SS scale were hypothesised to be more likely to exhibit BA. Our primary research questions were:

i. Do drivers change their car-following behaviour in manual driving after experiencing car-following in automated driving?

ii. Is this influenced by the THW adopted by the automated driving system?

iii. Is this influenced by whether drivers resume control in the presence of a lead vehicle?

iv. Is this influenced by engaging in a visual NDRT during automation?

\section{Methods}

\subsection{Participants}

Following approval from the University of Leeds Research Ethics Committee (Reference Number: LTTRAN-054), we recruited two groups of 16 drivers, via the driving simulator database. Participant details for each group are displayed in Table 1. Participants received $£ 25$ for taking part in the experiment and were free to withdraw at any point. Three participants were not considered for analysis, as they did not adhere to the experiment instructions to follow the lead vehicle. One participant was excluded because of missing data.

\subsection{Design and procedure}

\subsubsection{Equipment}

The experiment was conducted in the full motion-based University of Leeds Driving Simulator (UoLDS), which consists of a Jaguar S-type cab housed in a 4 m-diameter spherical projection dome with a $300^{\circ}$ field-of-view projection system. The simulator also incorporates an 8 degree-of-freedom

\begin{tabular}{llllll}
\hline Demographics & \multicolumn{2}{l}{ Gender group, mean $(\mathrm{SD})$} & & \multicolumn{2}{l}{ Automation group, mean (SD) } \\
\cline { 2 - 3 } & Males $(N=19)$ & Females $(N=9)$ & & L2 $(N=15)$ & L3 $(N=13)$ \\
\hline Age (years) & $39(16)$ & $38(10.83)$ & & $42(17)$ & $33(8)$ \\
Miles travelled annually & $11,368(9401)$ & $7763(4302)$ & & $8753(4719)$ & $9116(8200)$ \\
Years of driving experience & $19(15)$ & $16(8)$ & & $22(16)$ & $14(8)$ \\
\hline
\end{tabular}


electrical motion system. This consists of a $500 \mathrm{~mm}$ strokelength hexapod motion platform, carrying the $2.5 \mathrm{~T}$ payload of the dome and vehicle cab combination, and allowing movement in all six orthogonal degrees-of-freedom of the Cartesian inertial frame. Additionally, the platform is mounted on a railed gantry that allows a further $5 \mathrm{~m}$ of effective travel in surge and sway.

When active, the ADS assumed lateral and longitudinal vehicle control and maintained a maximum velocity of 40 $\mathrm{mph}$. However, in the presence of a slower lead vehicle, the system would reduce its speed, to maintain the time headway of the respective condition (described below). The status of the ADS was indicated by the colour of a steering wheel symbol that was located on the left panel of the central display unit (Fig. 1). During the automated drives, the steering wheel symbol was solid green when automation was engaged, and red when automation was unavailable.

\subsubsection{Experimental design}

A $2 \times 2 \times 2$ mixed design was used for this study, with a between-participants factor of Level of Automation (L2, L3) and within-participant factors of Time headway (Short: $0.5 \mathrm{~s}$, and Long: $1.5 \mathrm{~s}$ ) and Take-over type (with lead car, without lead car). All factors were fully counterbalanced.

Level of Automation determined the activities drivers were permitted to do during automated driving. Participants in the L3 group were instructed to engage in a visual nondriving-related "Arrows" task (NDRT) during automation (Jamson and Merat 2004). The Arrows task required participants to search for, and touch, the upward-facing Arrow, displayed in a $4 \times 4$ grid of Arrows, using a touch screen in the centre console. The screen displayed the current participant's cumulative score and a 'score to beat' to keep them engaged in the task. Participants were also told they would get an additional $£ 5$ if they beat the best score, though, for ethical reasons, all participants received this reward at the end of the experiment, regardless of performance. The Arrows task was only available when automation was engaged.
Take-over type specifies whether drivers resumed control during a car-following, or free-following, scenario. For all experimental drives, approximately 2 min after drivers engaged automation, a lead vehicle moved into the ego vehicle's lane, from an adjacent road, triggering automated carfollowing. However, for half of the trials, the lead vehicle continued in its path when the transition to manual control was triggered, while for the other half of the trials, the lead vehicle exited the lane a few moments before the take-over event (see Fig. 2). For the trials without a lead vehicle, a new lead vehicle joined the ego vehicle's lane, from an adjacent road at the next intersection, which was $20 \mathrm{~m}$ from the previous intersection. The aim of this manipulation was to assess whether, after the resumption of control, drivers would attempt to catch up, and then maintain the same headway with a new lead vehicle, as the headway assumed with a vehicle immediately ahead of them. Note that a late resumption of control never led to a crash, unless drivers sped up after resumption of control, since the lead vehicle always assumed a safe headway.

\subsubsection{Procedure}

Upon arrival, participants were briefed on the description of the study and were asked to sign a consent form, with an opportunity to ask any questions. They were then given a chance to practice manual driving, and automated driving, within a 2-lane urban road, with low-density oncoming traffic. During the practice session, participants were talked through the various aspects of the vehicle HMI, were shown how to engage and disengage the automation and, those in the L3 condition practiced the Arrows task.

Participants were asked to drive in the centre of the lane and maintain the $40 \mathrm{mph}$ speed limit. They were asked not to overtake any lead vehicles, but to otherwise adhere to the standard rules of the road, ensuring safe operation of the vehicle, and maintaining their desired distance to the vehicle ahead. Before the start of the automated drives, participants were presented with an auditory-verbal request to engage
Fig. 1 An example of the invehicle HMI with the automation status symbol (Left: automation not engaged, Right: automation engaged) and the vehicle speed (mph)

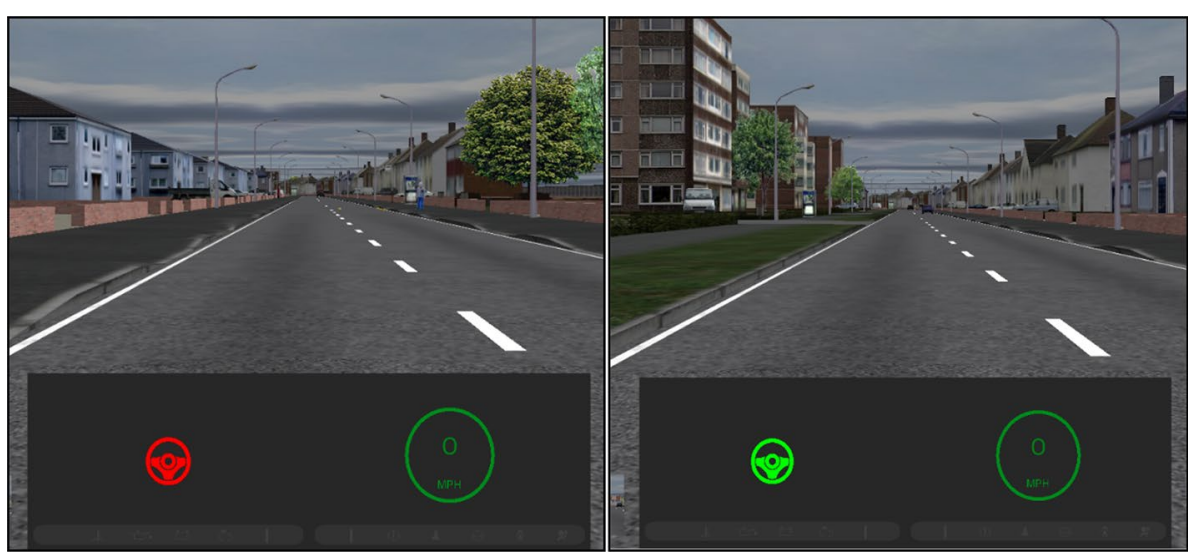


Experimental Run 1/2 ( 18 Minutes): Long/Short THW during automated car-following

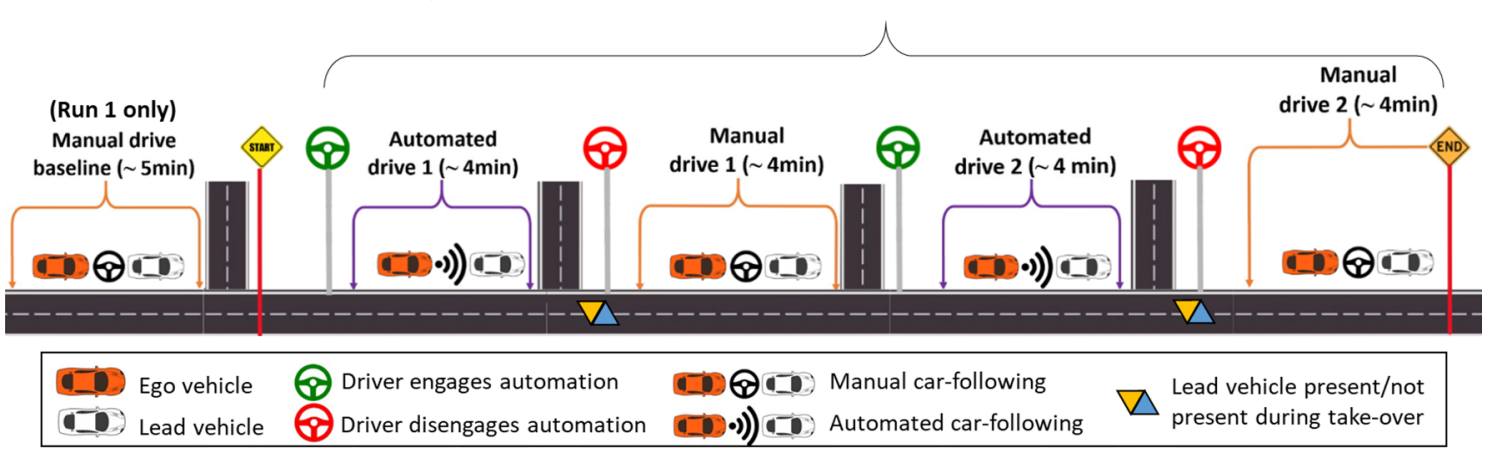

Fig. 2 Schematic representation of the two experimental runs, which exposed drivers to automated car-following with either a long $(1.5 \mathrm{~s})$ or short $(0.5 \mathrm{~s})$ time headway. Each run comprised of two sequences of automated and manual car-following drives. Only the first run

automation: "Attention engage automation". To engage the ADS, participants pressed a button on the steering wheel, after which they took their hands away from the steering wheel and foot away from the accelerator. At the end of the automated drives, participants were presented with an auditory-verbal take-over request, "Attention, get ready to takeover". The TOR was presented when the vehicle reached a section of road with faded road markings, which represented a system limitation condition, and a need to resume control. After this alert, a short-duration acoustic tone $(1000 \mathrm{~Hz}$, lasting $0.2 \mathrm{~s}$ ) sounded with increasing frequency until participants resumed manual control. Participants could disengage automation by either pulling the stalk, moving the steering wheel (threshold of $2^{\circ}$ was applied), or pressing the brake, or accelerator pedals. Our aim was to implement a non-critical take-over request that did not cause drivers any distress. The road markings reappeared shortly after drivers resumed control. All drivers resumed control, and the exact take-over time varied according to when drivers resumed control, but it was generally between 10 and $13 \mathrm{~s}$.

Following the practice drive, participants completed two experimental runs (see Fig. 2). Run 1 consisted of five different, but connected, driving segments, starting with a brief Manual Baseline Drive ( $6 \mathrm{~min}$ ) which started with a $\sim$-min free-driving scenario, and a $\sim 5$-min car-following scenario, after which the lead vehicle turned off the road and drivers carried on driving for $\sim 1 \mathrm{~min}$. This period was used to collect 'baseline' data for drivers' THW during carfollowing and was only included in Run 1.

Apart from Manual Baseline Drive, the sequence of events for Run 1 and Run 2 were identical. Each driver experienced the following order of events: Automated Drive 1 ( $\sim 5 \mathrm{~min})$, Manual Drive 1 ( $\sim 5 \mathrm{~min})$, Automated Drive 2 ( $\sim \mathrm{min})$, and Manual Drive 2 ( $\sim \mathrm{min})$. Run 2 began with a brief period of manual driving to allow participants to engage the ADS. Experimental run 1 and of the experiment included a manual baseline car-following drive. Between each drive, drivers had to take-over control, either with or without a lead vehicle. The order of the runs and presence of the lead vehicle during the take-over was counterbalanced across participants

2 were counterbalanced, which varied the order in which drivers experienced long and short THW automated carfollowing. Within each drive, whether or not drivers resumed control in the presence of a lead vehicle was also counterbalanced.

To reduce the effect of fatigue, a short break was introduced after the practice drive and experimental drives. After each of these drives, participants were taken out of the driving simulator, and asked to complete a three-part questionnaire, which included the Arnett Inventory of Sensation Seeking (AISS; Arnett 1994), traffic locus of control (T-LOC; Özkan and Lajunen 2005), and driver style questionnaires (DSQ; French, West, Elander and Wilding 1993). Finally, after Run 1 and Run 2, respectively, drivers rated their perceptions of their own and the ADS behaviour during the preceding drive (either Long THW or Short THW) by indicating on a five-point Likert scale (1: "Strongly disagree" to 5: "Strongly Agree") their level of agreement with the following statements:

i. During the automated drive, the system kept a safe distance from the car in front.

ii. During the automation drive, I think the system should have kept a closer distance from the car in front

iii. During the automated drive, I think the system should have kept a long distance from the car in front.

iv. Experiencing the automated driving system changed how I drove in the subsequent manual drive.

v. Following the automated drive, when there was a vehicle in front of me, I used the accelerator and brakes more than normal.

vi. I kept the same distance to the vehicle in front during the manual drive as I experienced in the automated drive.

The entire experiment lasted approximately $2.5 \mathrm{~h}$. 


\subsection{Analysis}

\subsubsection{Establishing car-following}

To analyse drivers' car-following behaviour, following resumption of manual control from automation, we first needed to establish that they had stabilised their control of the vehicle, and were engaged in a consistent car-following behaviour. The concept of stability in car-following was initially proposed by Herman et al. (1959) and is characterised as a consistent variation in drivers' following distance, which does not affect the overall microstructure of the surrounding traffic. We calculated the point at which drivers had entered a stable car-following period, labelled "stabilisation time", using an algorithm developed by Gonçalves et al. (2020). In this work, the metric was measured as the time between the take-over, and the point at which drivers' average THW remained below a particular threshold, for at least $10 \mathrm{~s}$. This threshold was based on inflexion points in the overall distribution of the THW during the whole car-following task, for each driver. We used this technique to calculate stabilisation time for both take-over types, i.e., irrespective of whether or not there was a lead vehicle during the take-over.

To establish car-following events for our analysis, we considered driving data from the stabilisation time to the moment the lead vehicle left the road. According to Gipps (1981), a car-following task is characterised by a constant mediation, and adjustment, of drivers' distance to the lead vehicle, according to their desired safety boundaries and willingness to increase their speed. Therefore, we filtered out the sections of manual driving when drivers were too far away from the lead vehicle for this mediation to happen. Since our scenario was in an urban environment, we only included events in which drivers had a THW lower than $6 \mathrm{~s}$. This was based on the method used by Vogel (2002), who found that a 6-s THW was the optimal threshold for distinguishing between free, and following vehicles, in urban environments.

\subsubsection{Statistical analyses}

We used Kolmogorov-Smirnov tests to test the normality of the data. Whenever the normality assumption was violated, we used logarithmic transformations to correct the observed positive skew, allowing the use of parametrical tests. If transformations were applied, the results of the statistical tests shown are based on the transformed data, but the plots and graphs are generated using the untransformed data.

We analysed data with SPSS V.24 (IBM, Armonk, New York, USA), and generated the visualisations in R. An $\alpha$-value of 0.05 was used as the criterion for statistical significance, and partial eta-squared was computed as an effect size statistic. Unless otherwise stated, variance of the data was homogenous, as assessed by Levene's test of equality of error variance. Similarly, following log transformation of the skewed data, covariance of the data was homogenous, as assessed by Box's test of equality of covariance matrices.

\section{Results}

\subsection{Mean time headway}

To understand the characteristics of the underlying car-following behaviour, we first plotted the THW distributions during car-following for each condition. Figure 3 shows all car-following events for the L2 and L3 groups, for the Baseline Manual Drive, and the manual drives after the Long and Short THW conditions, and Car and No Car conditions. The 6-s threshold we employed seemed to separate car-following from free-driving scenarios, as there were no outliers across the distributions. The THW distributions generally followed the distributions observed in other studies, with the exception of the Baseline Manual Drives and the post-automation drives in which drivers experienced a Long THW and resumed control when there was no lead vehicle. Here, longer THWs were generally observed compared to the other drives.

Since there was only one Baseline Manual Drive per participant, and four post-automation manual drives, it was not possible to assess, in a single step, whether there were changes in THW, after each automation drive. Therefore, the analysis of mean THW changes was conducted in two parts: first, we compared drivers' THW in the Baseline Manual Drive with each combination of Time headway and Take-over type conditions, using four separate $2 \times 2$ ANOVAs. For each analysis, we used a within-participant factor of Exposure to automation (Baseline Manual Drive, PostAutomation Drive), and a between-participant factor of Level of Automation (L2, L3). The Post-Automation Drive was based on the specific combination of conditions drivers were exposed to. For example, if they resumed control in the presence of a lead vehicle after a Short THW condition, this is referred to as "Car+Short". The same applies to the other condition combinations: "No Car + Short", "Car + Long", and "No Car + Long".

Second, to understand whether changes were influenced by any of the experimental conditions, we calculated the difference in THW between Baseline Manual Drive and the post-automation manual drives, and then compared these using a $2 \times 2 \times 2$ mixed ANOVA. The within-participant factors were time headway during automation (Short, Long) and Presence of lead vehicle during Take-over (Car, No Car), and the between-participant factor was Automation condition (L2, L3). This was used to investigate whether drivers changed their THW after being exposed to automation, and 


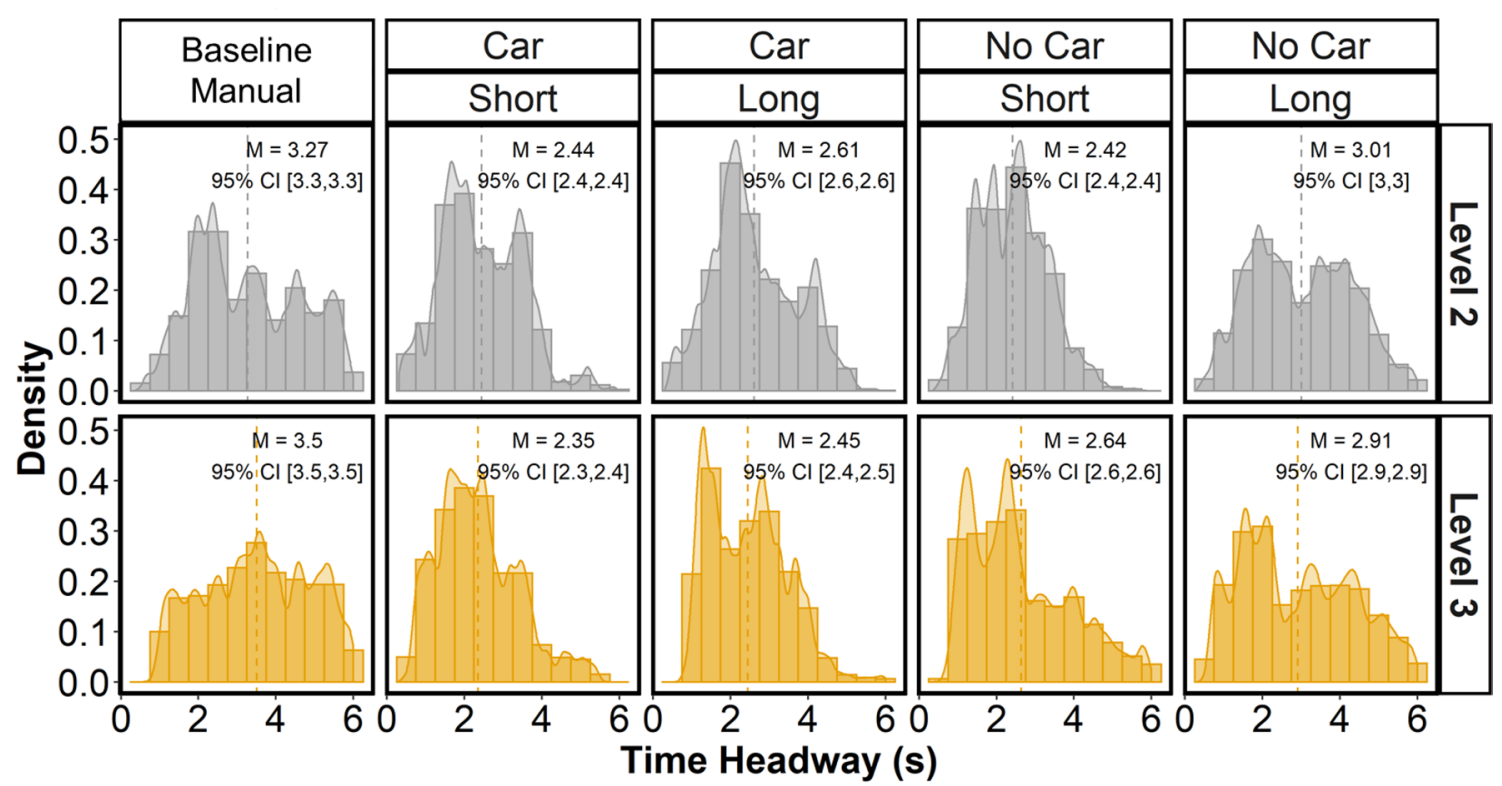

Fig. 3 Time headway distribution for the L2 and L3 groups, for the Baseline Manual Drive and each of the Long and Short conditions, and Car and No Car conditions. Vertical dashed lines represent the distribution mid-points

if the conditions influenced the magnitude of this change. Initially, we included each of the subscales of AISS, T-LOC, and DSQ as covariates in the ANOVAs. However, all of these subscales returned non-significant effects and small effect sizes. Therefore, to maintain statistical power, these covariates were removed from the analyses.

The first set of ANOVAs we conducted revealed that drivers' THW in the Baseline Manual Drive was significantly higher, compared to all subsequent post-automation manual drives (Fig. 4). On average, in the Baseline Manual
Drive, drivers had a THW of $3.78 \mathrm{~s}$, whereas the global mean for all post-automation car-following events was $2.7 \mathrm{~s}$. In other words, absolute THW during car-following decreased significantly after experiencing automated car-following. Across all ANOVAs, there was no effect of Level of Automation and no interactions, which suggests that the reduction in THW occurred irrespective of whether drivers were engaged in an NDRT during automation (L3), or were looking around the road environment during automation (L2).
Fig. 4 Mean time headway (s) during manual car-following during the Manual Baseline Drive and the four post-automation manual drives. $* * p<.005$ $* * * p<.001$

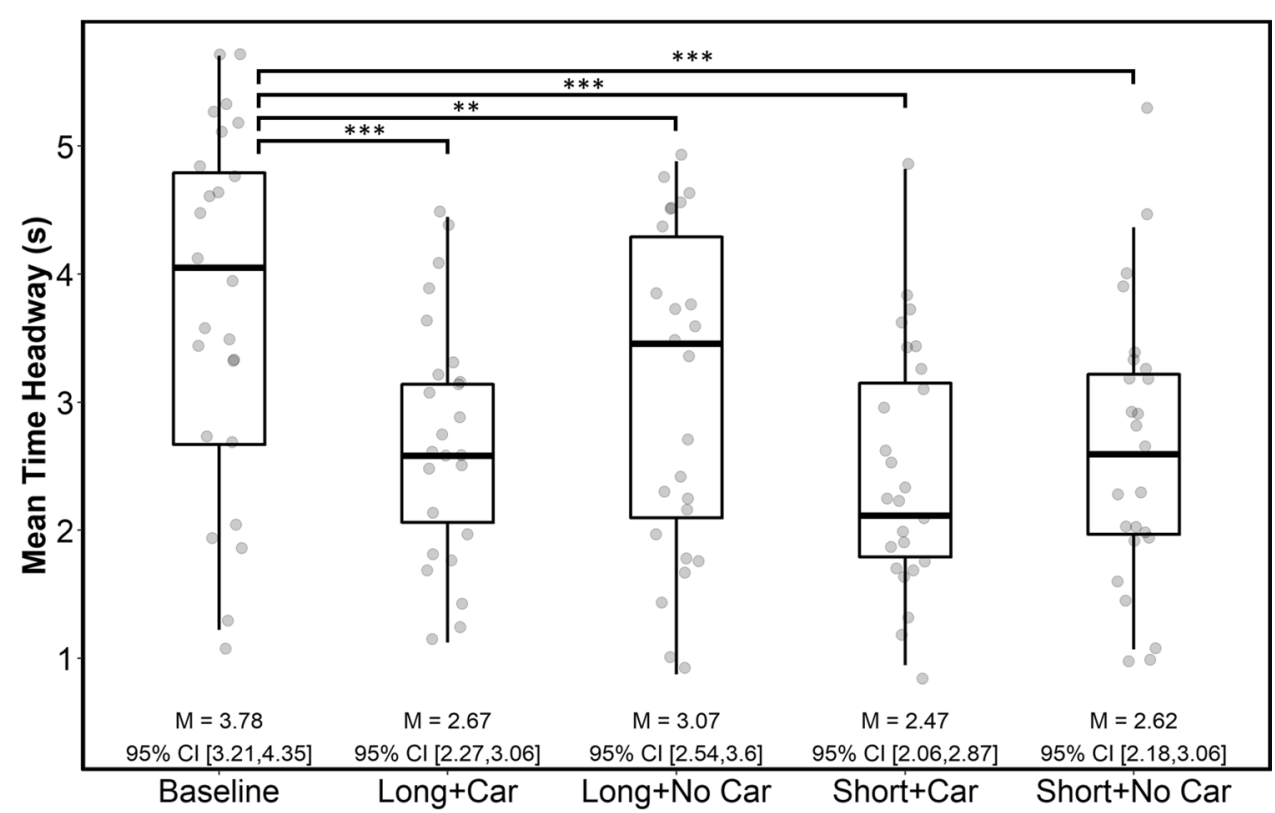


The second ANOVA revealed that there was a main effect of length of Time headway during automation $\left(\mathrm{F}(1,23)=4.320, p<0.05, \eta_{\mathrm{p}}{ }^{2}=0.158\right)$ on how much drivers changed their THW, compared to their Baseline Manual Drive. As shown in Fig. 5a, drivers had significantly shorter THWs during the post-automation manual car-following, after the Short THW conditions (M: $-1.25 \mathrm{~s}$ ), compared to after the Long THW conditions (M: $-0.9 \mathrm{~s}$ ). Therefore, there was an immediate effect of the set THW during automation, on drivers' subsequent adopted headway. There was also a main effect of presence of lead vehicle during take$\operatorname{over}\left(\mathrm{F}(1,23)=11.339, p<0.01, \eta_{\mathrm{p}}{ }^{2}=0.330\right)$, where Fig. $5 \mathrm{~b}$ shows THW during post-automation car-following appeared to reduce significantly more, relative to the Baseline Manual Drive, if drivers resumed control in the presence of a lead vehicle (M: $-1.23 \mathrm{~s}$ ) compared to resuming control without a lead vehicle (M: $-0.92 \mathrm{~s}$ ). In other words, we know that drivers reduce their THW during car-following after experiencing automated car-following, but the reduction is more pronounced if drivers resume control during a carfollowing event, rather than restarting a car-following event a little later. These results suggested that drivers were not only mimicking the THW they had just experienced, but the effect was more pronounced when the car-following event persisted through the resumption of control.

There was no effect of automation level $(\mathrm{F}(1,23)=0.006$, $\left.p=0.999, \eta_{\mathrm{p}}{ }^{2}=0.000\right)$ and no interactions, which suggests that monitoring the environment and observing the THW during automated car-following (L2) did not influence the extent to which drivers reduced their THW. While the NDRT in the L3 group was designed to take drivers' visual attention away from the forward path, it is possible that they made short glances to the road during automation. In this case, the results suggests that ADS use can influence drivers' behaviour, even if they are not fully aware of, or continuously monitoring, its performance. In addition, all drivers were exposed to the lead vehicle for a short period immediately after the TOR, which may also have influenced their subsequent adopted headway.

\subsection{Standard deviation of time headway}

One of the primary concerns about the effect of vehicle automation on drivers' behaviour is the extent to which it affects their control of the vehicle, once they resume manual control. Mean THW is a useful measure for understanding the degree of risk that drivers are willing to accept during car-following. However, equally important, from a controllability standpoint, is the steadiness or consistency with which drivers control their vehicle after automation. During carfollowing, this would be reflected by the variation in drivers' THW, which also indicates drivers' 'safety boundary' (Boer 1999). To examine whether there were changes to the variation in drivers' THW, we followed the same two stages of analysis described above. First, we compared drivers' standard deviation (SD) of THW in the Baseline Manual Drive with each combination of Time headway and Take-over type, using four separate $2 \times 2$ ANOVAs, with a within-participant factor of Exposure to automation (Baseline Manual Drive, Post-Automation Drive - as described in the previous section), and a between-participant factor of Level of Automation (L2, L3). Second, to understand whether changes
Fig. 5 Difference in mean time headway (s) during car-following between the Baseline Manual Drive and post-automation drives, for the conditions where a drivers experienced Long or Short THW during automated car-following, and where b drivers resumed control with a lead car (Car) or without a lead car (No Car). The red dotted line represents the Baseline Manual Drive for all drivers. $* p<.05$ $* * p<.01$
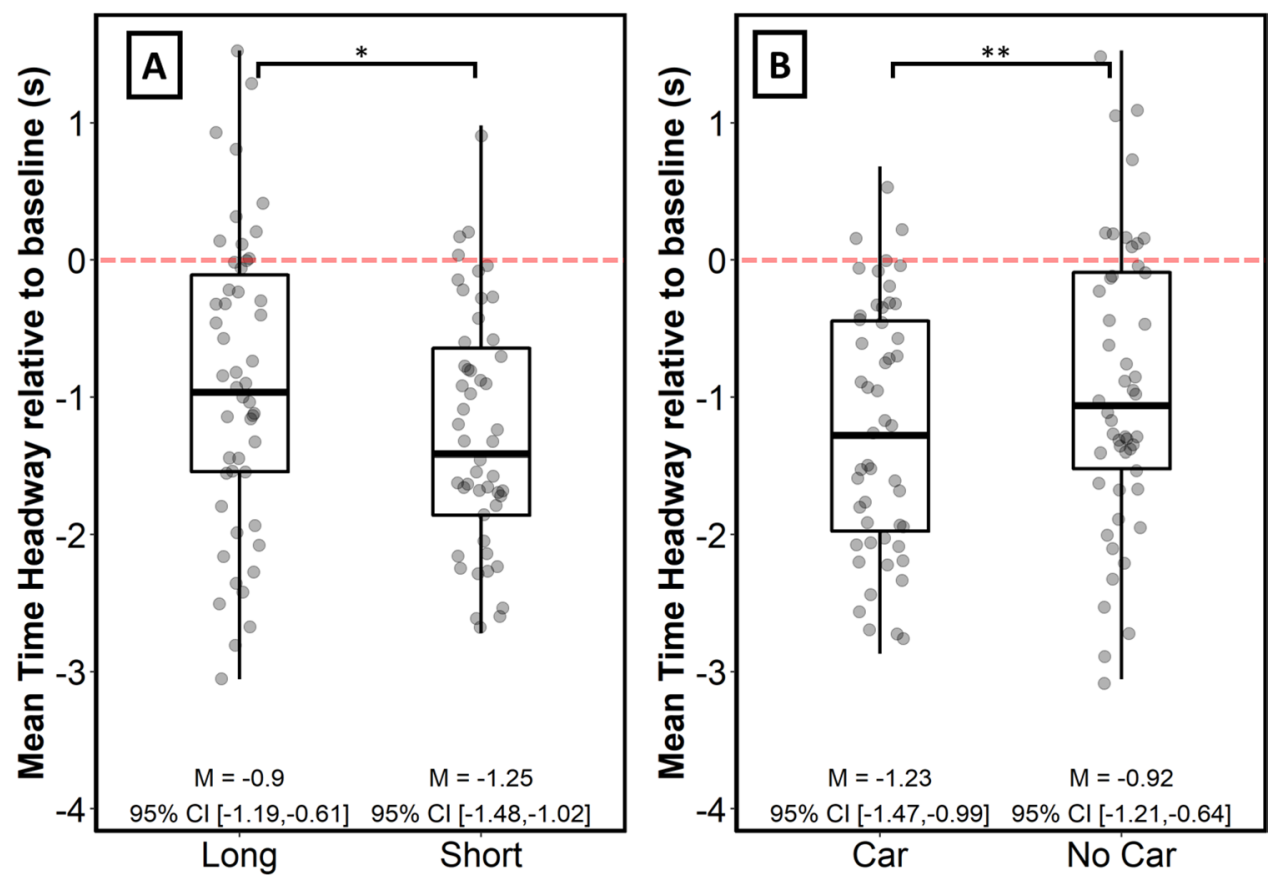
were influenced by the Time headway and Take-over type, we calculated the difference in SD of THW between Baseline Manual Drive and the respective conditions, comparing these with a $2 \times 2 \times 2$ ANOVA. The within-participant factors were Time headway during automation (Short, Long) and Presence of lead vehicle during Take-over (Car, No Car), and the between-participant factor was Automation condition (L2, L3).

Across all four 2X2 ANOVAs, comparing SD of THW in the Baseline Manual Drive to post-automation manual drives, there was no effect of Exposure to automation, no effect of Level of Automation, and no interactions (Fig. 6). These results indicate that, while drivers may have reduced their THW in car-following after automated car-following, their behaviour was quite consistent across the different conditions.

The second ANOVA revealed no effects of Exposure to Automation, Presence of lead vehicle during Take-over, Level of Automation, and no interactions, which is not surprising given that absolute SD of THW of each condition did not differ significantly, compared to Baseline Manual Drive. This indicates that THW variability was not influenced by whether or not drivers had their eyes away from the forward roadway during automation.

\subsection{Subjective assessment}

In addition to the T-LOC, AISS, and DSQ questionnaires, drivers were asked to provide a subjective assessment of the ADSs' behaviour, after the automated car-following drives with Long and Short THW (top three questions in Fig. 7).
Drivers were also asked to assess changes in their behaviour after each of these drives (bottom three questions in Fig. 7).

$76 \%$ of drivers felt that the ADS kept a safe distance from the car in front, during the Long THW condition, while 84\% of drivers disagreed with this statement for the Short THW condition. For both the Long and Short THW conditions, the majority of drivers ( $84 \%$ and $92 \%$, respectively) did not feel that the ADS should have kept a closer distance from the car in front. However, there was more consensus across drivers that the ADS should have kept a longer distance to the lead vehicle, for the Short THW condition. These responses suggest that drivers were able to differentiate between the experimental conditions, and while the Long THW condition was generally tolerable, the Short THW was viewed as unsafe.

There was no clear agreement between drivers about whether they had changed their behaviour after using the ADS, though most drivers felt that they did not use the brakes and accelerator pedals more after the automated drives. Given that drivers assessed the Short THW condition to be unsafe, it is unsurprising that $92 \%$ indicated that they did not keep the same distance to the lead vehicle in the subsequent manual drive.

To determine whether what drivers subjective response in terms of their perceived behaviour after automation was reflected in their actual behaviour, we ran two separate Pearson product-moment correlations of drivers' responses, comparing response to the item "Experiencing the automated driving system changed how I drove in the subsequent manual drive" with their actual mean THW. Post automation THW was compared to Baseline Manual Drive values, for
Fig. 6 Standard deviation of time headway (s) during manual car-following for the Baseline Manual Drive and the four postautomation manual drives

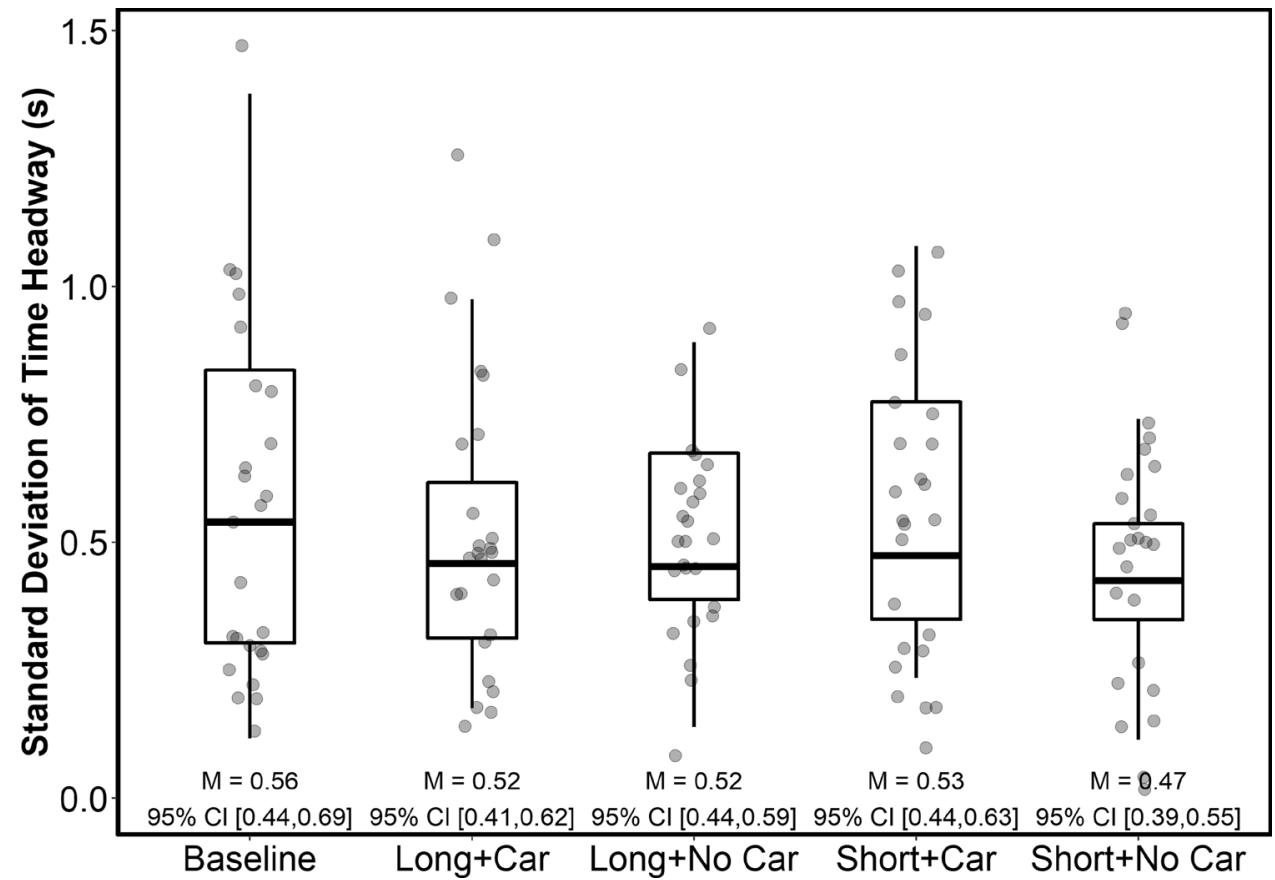




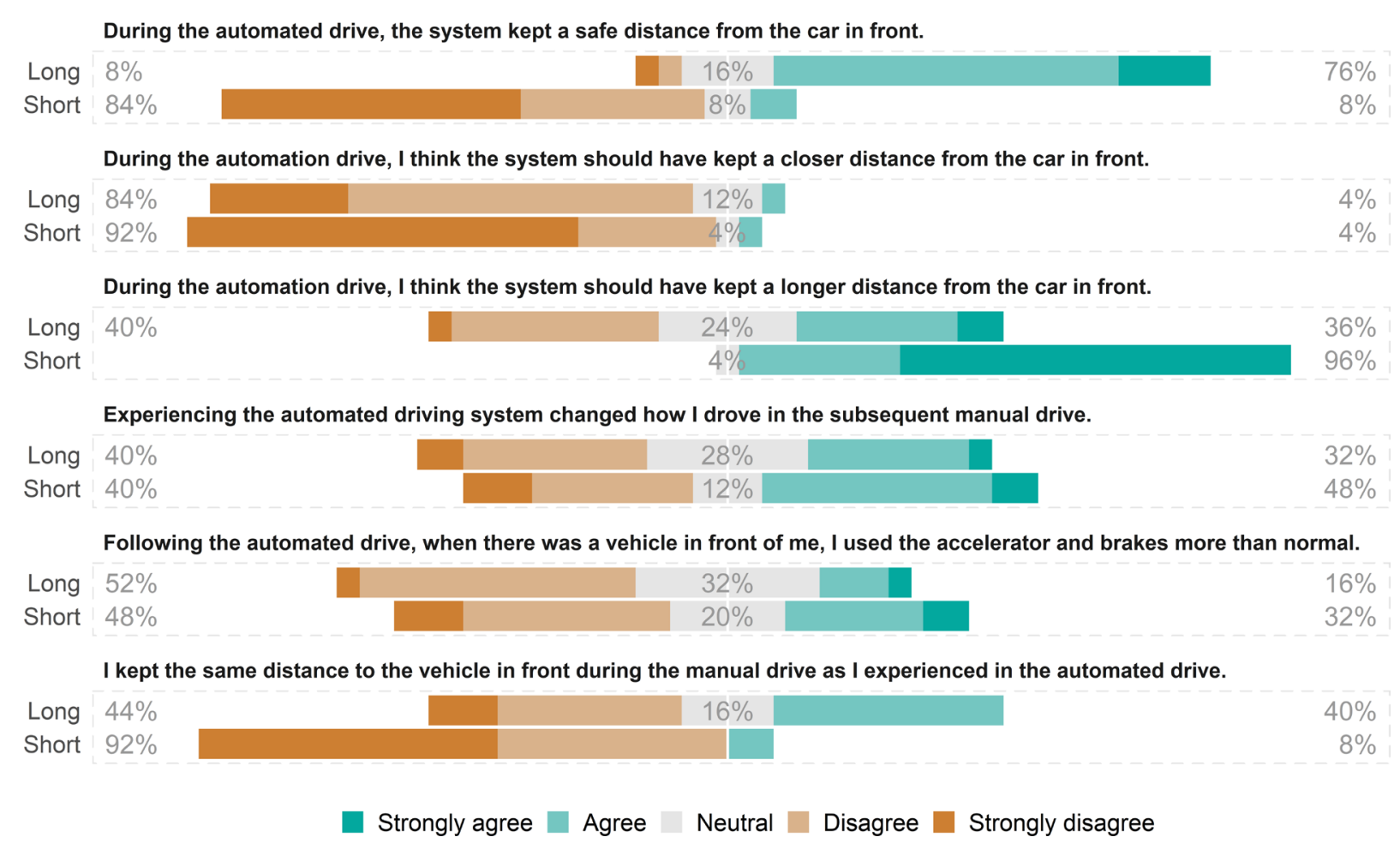

Fig. 7 Drivers' subjective assessment of the ADSs' behaviour during automated car-following, and their judgement of their own behaviour during post-automation manual driving

both the Long and Short THW conditions, while also controlling for the Level of Automation.

There was a moderate, negative significant correlation between the two measures for the Long THW condition $(r(47)=-0.341, p=0.016)$, showing that what drivers thought they did was opposite to what they actually did. However, there was no significant association in the Short THW condition $(r(47)=-0.010, p=0.944)$. Therefore, drivers' assessment of their behaviour did not match their actual behaviour.

\section{Discussion and conclusions}

This driving simulator study assessed changes in driver's manual car-following behaviour after automated car-following in an urban environment. The study had two experimental groups: during automated car-following, one group was engaged in an NDRT (L3), while the other group was free to look around the road environment (L2). We also compared the effect of Long (1.5 s) and Short (0.5 s) THW conditions during automated car-following, and whether the presence of a lead vehicle, during the resumption of control, had an impact on any subsequent changes in car-following behaviour. All post-automation drives were compared to a
Baseline Manual Drive, which was recorded at the start of the experiment.

As our first research question, we sought to understand whether drivers change their car-following behaviour in manual driving after experiencing car-following in automated driving. Our results showed that drivers significantly reduced their time headway in all post-automation drives, compared to a Baseline Manual Drive. This is in line with the findings of both Skottke et al. (2014), Eick and Debus (2005), who showed that drivers reduced their time headway after being decoupled from highly automated driving and truck platoons. This pattern has also been observed in a study on drivers' behavioral adaptation after using full-range ACC (Varotto et al. 2020). This can be explained through risk homeostasis theory, where, as drivers' become more familiar and comfortable with shorter THWs during automated driving, they adjust their boundary of acceptable risk. In other words, drivers become used to following at shorter distances with no negative outcomes, despite not being in control of the vehicle. However, as drivers' resume manual control, this adapted risk boundary carries over into their own manual driving, and they accept shorter THWs than they otherwise would. The observed changes in behaviour justify our concern regarding the potential increased susceptibility to rear-end collisions after automated driving, as shorter THWs increase the risk of rear-end collisions (Lee, 
Llaneras, Klauer and Sudweeks 2007). Future research should confirm our results and examine the extent to which adaptation of car-following behaviour after automated driving impacts drivers' abilities to respond in such situations.

Our second and third research questions addressed whether any changes in post-automation car-following were influenced by the THW adopted by the automated driving system and whether drivers resumed control in the presence of a lead vehicle. Our results showed that there was a greater reduction in THW after drivers resumed control in the presence of a lead vehicle, and also after they had experienced a shorter THW $(0.5 \mathrm{~s})$ during automated car-following. These results demonstrate that the THW drivers adopt in manual car-following is influenced by the THW they were exposed to during automated car-following, especially if the carfollowing event persists through the resumption of control. While shorter THWs adopted by automated vehicles may lead to optimised traffic flow and capacity (Friedrich 2016), our results suggest that this should be carefully balanced against the potential negative impact this will have on drivers' manual driving behaviour, as well as their acceptance and, ultimately, use of the system.

For our final research question, we sought to understand whether any changes in post-automation car-following would be influenced by whether or not drivers' engaged in a visual NDRT during automation. We found that there were no differences in THW changes between the L2 and L3 groups, suggesting that drivers do not need to continuously monitor the road environment for their THW to be influenced by the ADS behaviour. It could be that during L3 driving, drivers perceived the lead vehicle via peripheral vision, possibly reinforced by short glances to the roadway. However, future research should clarify this hypothesis.

Based on research by Itkonen and Lehtonen (2020) and Rudin-Brown and Parker (2004), another aim of this research was to investigate whether any changes in behaviour were associated with drivers' self-reported traits, including sensation seeking (AISS, Arnett 1994), traffic locus of control (T-LOC, Özkan and Lajunen 2005), and driver style questionnaire (DSQ, French, West, Elander and Wilding 1993). However, the changes in THW we observed did not appear to be associated with any subscales of the T-LOC, AISS, or DSQ questionnaires, suggesting that the changes observed here may not be linked to the underlying personal traits we investigated. These results are in contrast to previous work on the link between individual characteristics such as sensation seeking and locus of control on behaviour changes (Ward, Fairclough, and Humphreys 1995; Rudin-Brown and Parker 2004).

In addition to the above, we sought drivers' perceptions of their own and the ADS behaviour during car-following. Drivers' subjective responses showed that their change in behaviour was not necessarily reflected in their subjective assessment of their behaviour change. That is, drivers were not aware that they had changed their behaviour, even in the short THW condition, which they overwhelmingly rated as unsafe. This is not surprising, as previous studies have shown that individuals are not always aware of how the use of technology can change their behaviour; for example, the effect negative effect of using a mobile phone while driving on performance and mental processing (Boase, Hannigan and Porter 1988; Alm and Nilsson 1995).

\subsection{Recommendations}

Our research highlights a number of areas that can be addressed to limit the adverse effect of BA to automation on manual driving. First, the system in use should be designed in a way that limits negative BA. For example, it is clear from drivers' behavioural change in the current study that the system should have adopted a more conservative THW. Second, drivers should receive explicit training about the potential effects that automation use may have on their manual driving, so that they do not become complacent. For example, if, in the current study, drivers were warned that their THW might shorten after using automation, it may have reduced the likelihood that this occurred. Third, drivers should be warned when their behaviour exceeds certain safe boundaries of operation. For example, in the current study, drivers could have been warned during manual driving that their THW had shortened compared to either their normal driving style or a safe standard. Indeed, some authors have already proposed some solutions; for example, Vanderhaegen (2016) developed and validated a rule-based system that assists drivers in dissonance discovery while using an Automated Speed Control System (ASCS).

\subsection{Limitations}

We should note that the THWs adopted by drivers in the first manual drive of this experiment are longer than what is commonly observed during real-world car-following. This may be due to drivers' unfamiliarity with the driving simulator and the urban road environment they were travelling in. If the THW observed in the Baseline Manual Drive is higher than that adopted by our participants in real-world driving, it may partially account for the reduction in postautomation THW. However, this would not account for the differences observed between the Long and Short THW conditions, or the Car and No Car conditions. Moreover, Vogel (2002) found that 6-s THW is an optimal threshold for distinguishing between free and following vehicles in urban environments, suggesting that the behaviour we observed in this study can be considered to be car-following and not free-driving. In addition, the experimental drives used in this study were relatively short, and though it is interesting 
to note that behavioural adaptations may exist after such a short period, this may not necessarily represent the realworld pattern system usage. For example, we did not consider the impact that fatigue and hypovigilance may have had on drivers' attention to the car-following task during automated driving, and, therefore, on their car-following behaviour in subsequent manual car-following.

\subsection{Future work}

Notwithstanding the above concerns, the trends observed here are generally in line with those of Skottke et al. (2014) and Eick and Debus (2005), who found that drivers reduced their THW for periods in manual driving, after decoupling from fully automated driving. However, it is an open question whether the kinds of changes we observed here would be seen after the use of ADS in daily use. For example, how does drivers' behaviour change after using ADF over more extended periods, such as weeks or months? It is also important to consider whether behavioural adaptations are consistent across different settings, for example, on motorways, rural roads, and urban environments. Furthermore, are there behavioural adaptations after using ADS in different use-cases, such as lane changes, parking, or merging? There is also merit in investigating whether the type of take-overs (i.e., critical vs non-critical; Erikson and Stanton 2017) influence the extent to which behavioural adaptation carries over into subsequent manual driving. Finally, how does behaviour adapt after different usage patterns, for example, less frequent, but more extended periods vs more frequent, but shorter periods, as previous research has shown that regular use of cruise control, for example, can lead to a reduction in vigilance and increase in reaction time (Dufour 2014). Therefore, future research should endeavour to investigate these issues, as ADS use will become more widespread in the coming years, and it is imperative that we understand the prospective risks of using ADAS and ADS.

\subsection{Conclusions}

Our results build on the research into behavioural adaptation and ADAS use and show that there is the potential for drivers' behaviour to adapt negatively after using automated driving systems, during car-following. In the coming decades, humans will likely be still involved in the driving task to varying degrees, so it is important from a safety perspective to understand what issues there are and for researchers and vehicle manufacturers to develop appropriate countermeasures.

Acknowledgements The research leading to these results has received funding from the European Commission Horizon 2020 program under the project L3Pilot, grant agreement number 723051. Responsibility for the information and views set out in this publication lies entirely with the authors. The authors would like to thank all partners within L3Pilot and the University of Leeds Driving Simulator for their cooperation and valuable contribution.

Open Access This article is licensed under a Creative Commons Attribution 4.0 International License, which permits use, sharing, adaptation, distribution and reproduction in any medium or format, as long as you give appropriate credit to the original author(s) and the source, provide a link to the Creative Commons licence, and indicate if changes were made. The images or other third party material in this article are included in the article's Creative Commons licence, unless indicated otherwise in a credit line to the material. If material is not included in the article's Creative Commons licence and your intended use is not permitted by statutory regulation or exceeds the permitted use, you will need to obtain permission directly from the copyright holder. To view a copy of this licence, visit http://creativecommons.org/licenses/by/4.0/.

\section{References}

Alm H, Nilsson L (1995) The effects of a mobile telephone task on driver behaviour in a car following situation. Accid Anal Prev 27:5

Arnett J (1994) Sensation seeking: A new conceptualisation and a new scale. Person Individ Differ 16(2):289-296

Boase M, Hannigan S, Porter JM (1988) Sorry, can’t talk now... just overtaking a lorry: The definition and experimentation investigation of the problem of driving and hands free car phone use. In Megaw ED (Ed) Contemporary ergonomics, London: Taylor and Francis, UK

Boer ER (1999) Car following from the driver's perspective. Transp Res Part F Traffic Psychol Behav 2(4):201-206

Brackstone M, Waterson B, McDonald M (2009) Determinants of following headway in congested traffic. Transp Res Part F Traffic Psychol Behav 12(2):131-142

Carsten O, Lai FC, Barnard Y, Jamson AH, Merat N (2012) Control task substitution in semiautomated driving: Does it matter what aspects are automated? Hum Factors 54(5):747-761

de Waard D, van der Hulst M, Hoedemaeker M, Brookhuis KA (1999) Driver behavior in an emergency situation in the automated highway system. Transp Human Factors 1(1):67-82

De Winter JC, Happee R, Martens MH, Stanton NA (2014) Effects of adaptive cruise control and highly automated driving on workload and situation awareness: A review of the empirical evidence. Transp Res Part F Traffic Psychol Behav 27:196-217

Dingus TA, Klauer SG, Neale VL, Petersen A, Lee SE, Sudweeks J, Bucher C (2006) The 100-car naturalistic driving study, Phase IIresults of the 100-car field experiment (No. DOT-HS-810-593). United States. Department of Transportation. National Highway Traffic Safety Administration

Dufour A (2014) Driving assistance technologies and vigilance: impact of speed limiters and cruise control on drivers' vigilance. Seminar on the impact of distracted driving and sleepiness on road safety, April. Paris La Défense

Eick EM, Debus G (2005) Adaptation effects in an automated car following scenario. Theory and application, Traffic and transport psychology, pp 243-255

Eriksson A, Stanton NA (2017) Takeover time in highly automated vehicles: noncritical transitions to and from manual control. Hum Factors 59(4):689-705

Evans L (1991) Traffic Safety and the Driver. Van Nostrand Reinhold, New York 
Evans L, Wasielewski P (1983) Risky driving related to driver and vehicle characteristics. Accid Anal Prev 15(2):121-136

French DJ, West RJ, Elander J, Wilding JM (1993) Decision-making style, driving style, and self-reported involvement in road traffic accidents. Ergonomics 36(6):627-644

Friedrich B (2016) The effect of autonomous vehicles on traffic. In Autonomous Driving (pp. 317-334). Springer, Berlin

Gipps PG (1981) Behavioral car-following model for computer simulation. Transp Res Part B Methodol 15(2):105-111

Heikoop DD, de Winter JC, van Arem B, Stanton NA (2019) Acclimatising to automation: Driver workload and stress during partially automated car following in real traffic. Transp Res Part F Traffic Psychol Behav 65:503-517

Herman R, Montroll EW, Potts RB, Rothery RW (1959) Traffic dynamics: analysis of stability in car following. Oper Res 7(1):86-106. https:// doi.org/10.1287/opre.7.1.86

Hoedemaeker M, Brookhuis KA (1998) Behavioural adaptation to driving with an adaptive cruise control (ACC). Transp Res Part F Traffic Psychol Behav 1(2):95-106

Itkonen TH, Lehtonen E (2020) Characterisation of motorway driving style using naturalistic driving data. Transp Res Part F Traffic Psychol Behav 69:72-79

Kulmala R, Rämä P (2013) Definition of behavioural adaptation. In: Rudin-Brown C, Jamson S (eds.), Behavioural adaptation and road safety: Theory, evidence and action (pp. 20). CRC Press

Larsson AF, Kircher K, Hultgren JA (2014) Learning from experience: Familiarity with ACC and responding to a cut-in situation in automated driving. Transp Res Part F Traffic Psychol Behav 27:229-237

Lee JD, Moray N (1994) Trust, self-confidence, and operators' adaptation to automation. Int J Hum Comput Stud 40(1):153-184

Lee SE, Llaneras E, Klauer SG, Sudweeks J (2007) Analyses of rear-end crashes and near-crashes in the 100-Car naturalistic driving study to support rear-signaling countermeasure development. (Report number DOT HS 810 846). Washington, DC: U.S. Department of Transportation

Loulizi A, Bichiou Y, Rakha H (2019) Steady-state car-following time gaps: an empirical study using naturalistic driving data. J Adv Transport

Louw T, Merat N (2017) Are you in the loop? Using gaze dispersion to understand driver visual attention during vehicle automation. Transp Res Part C Emerg Technol 76:35-50

Louw T, Markkula G, Boer E, Madigan R, Carsten O, Merat N (2017a) Coming back into the loop: Drivers' perceptual-motor performance in critical events after automated driving. Accid Anal Prev 108:9-18

Louw T, Madigan R, Carsten O, Merat N (2017b) Were they in the loop during automated driving? Links between visual attention and crash potential. Injury Preven 23(4):281-286

Lyons JB, Ho NT, Koltai KS, Masequesmay G, Skoog M, Cacanindin A, Johnson WW (2016) Trust-based analysis of an Air Force collision avoidance system. Ergonom Design 24(1):9-12

Lyu N, Deng C, Xie L, Wu C, Duan Z (2019) A field operational test in China: Exploring the effect of an advanced driver assistance system on driving performance and braking behavior. Transp Res Part F Traffic Psychol Behav 65:730-747

Merat at al (2014) Transition to manual: Driver behaviour when resuming control from a highly automated vehicle. Transp Res Part F Traffic Psychol Behav 27:274-282

Metz B, Wörle J, Hanig M, Schmitt M, Lutz A (2020) Repeated usage of an L3 motorway chauffeur: change of evaluation and usage. Information 11(2): 114

NHTSA (2009): A compilation of motor vehicle crash data from the fatality analysis reporting system and the general estimates system, U.S. Department of Transportation, DOT HS 811 402, Washington, DC

Niels F, Edoardo P, Florent GF, Clément V (2019) A new methodology to model driver behaviour accounting for the variation in driving manner using naturalistic driving data. In Proceedings: 26th International
Technical Conference on The Enhanced Safety of Vehicles. Eindhoven, Netherlands

Nilsson L (1995) "Safety effects of adaptive cruise controls in critical traffic situations", In: VERTIS (Ed.), Proceedings of Steps Forward, the Second World Congress on Intelligent Transport Systems, Vol III, 1294-9. Yokohama, Japan

Özkan T, Lajunen T (2005) Multidimensional traffic locus of control scale (T-LOC): factor structure and relationship to risky driving. Personal Individ Differ 38(3):533-545

Pekkanen J, Lappi O, Itkonen TH, Summala H (2017) Task-difficulty homeostasis in car following models: experimental validation using self-paced visual occlusion. PLoS One, 12(1)

Piccinini GFB, Rodrigues CM, Leitão M, Simões A (2014) Driver's behavioral adaptation to adaptive cruise control (ACC): The case of speed and time headway. J Safety Res 49:77-e1

Rudin-Brown C, Jamson S (2013) Behavioural adaptation and road safety: Theory, evidence and action. CRC Press

Rudin-Brown CM, Noy YI (2002) Investigation of behavioral adaptation to lane departure warnings. Transp Res Rec 1803(1):30-37

Rudin-Brown CM, Parker HA (2004) Behavioural adaptation to adaptive cruise control (ACC): implications for preventive strategies. Transp Res Part F Traffic Psychol Behav 7(2):59-76

Saifuzzaman M, Zheng Z (2014) Incorporating human-factors in carfollowing models: a review of recent developments and research needs. Transp Res Part C Emerg Technol 48:379-403

Salter RJ (1974) Headway distributions in highway traffic flow. In Highway Traffic Analysis and Design (pp. 107-124). Palgrave, London

Shen S, Neyens DM (2017) Assessing drivers' response during automated driver support system failures with non-driving tasks. J Safety Res 61:149-155

Siebert FW, Oehl M, Bersch F, Pfister HR (2017) The exact determination of subjective risk and comfort thresholds in car following. Transp Res Part F Traffic Psychol Behav 46:1-13

Skottke EM, Debus G, Wang L, Huestegge L (2014) Carry-over effects of highly automated convoy driving on subsequent manual driving performance. Hum Factors 56(7):1272-1283

Smiley A (2000) Behavioral adaptation, safety, and intelligent transportation systems. Transp Res Rec 1724(1):47-51

Stanton NA, Young M, McCaulder B (1997) Drive-by-wire: The case of driver workload and reclaiming control with adaptive cruise control. Saf Sci 27(2-3):149-159

Sullivan JM, Flannagan MJ, Pradhan AK, Bao S (2016) Literature review of behavioral adaptations to advanced driver assistance systems. AAA Foundation for Traffic Safety, Washington, D.C.

SAE (2018) Taxonomy and definitions for terms related to driving automation systems for on-road motor vehicles. Standard J3016_201806, Rev 2018-06-15. SAE International

Trimpop RM (1996) Risk homeostasis theory: problems of the past and promises for the future. Saf Sci 22(1-3):119-130

Van Winsum W (1998) Preferred time headway in car-following and individual differences in perceptual-motor skills. Percept Mot Skills 87(3):863-873

Vanderhaegen F (2016) A rule-based support system for dissonance discovery and control applied to car driving. Expert Syst Appl 65:361-371

Victor TW, Tivesten E, Gustavsson P, Johansson J, Sangberg F, Ljung Aust M (2018) Automation expectation mismatch: incorrect prediction despite eyes on threat and hands on wheel. Hum Factors 60(8):1095-1116

Vogel K (2002) What characterises a "free vehicle" in an urban area? Transp Res Part F Traffic Psychol Behav 5(1):15-29

Vogel K (2003) A comparison of headway and time to collision as safety indicators. Accid Anal Prev 35(3):427-433

Varotto SF, Farah H, Bogenberger K, van Arem B, Hoogendoorn SP (2020) Adaptations in driver behaviour characteristics 
during control transitions from full-range Adaptive Cruise Control to manual driving: an on-road study. Transp A: Transp Sci 16(3):776-806

Ward NJ, Fairclough S, Humphreys M (1995) The effect of task automatisation in the automotive context: a field study of an autonomous intelligent cruise control system. In: Garland DJ, Endsley MR (eds), Experimental analysis and measurements of situational awareness. Embry-Riddle Aeronautical University Press, Daytona Beach, Florida, USA, 369-374

Wilde GJS (1994) Risk homeostasis theory and its promise for improved safety. In: Trimpop RM, Wilde GJS (eds) Challenges to accident prevention: The issue of risk compensation behavior. Styx Publications, Groningen, The Netherlands, pp 9-24

Zhang B, de Winter J, Varotto S, Happee R, Martens M (2019) Determinants of take-over time from automated driving: A meta-analysis of 129 studies. Transp Res Part F Traffic Psychol Behav 64:285-307

Publisher's Note Springer Nature remains neutral with regard to jurisdictional claims in published maps and institutional affiliations. 